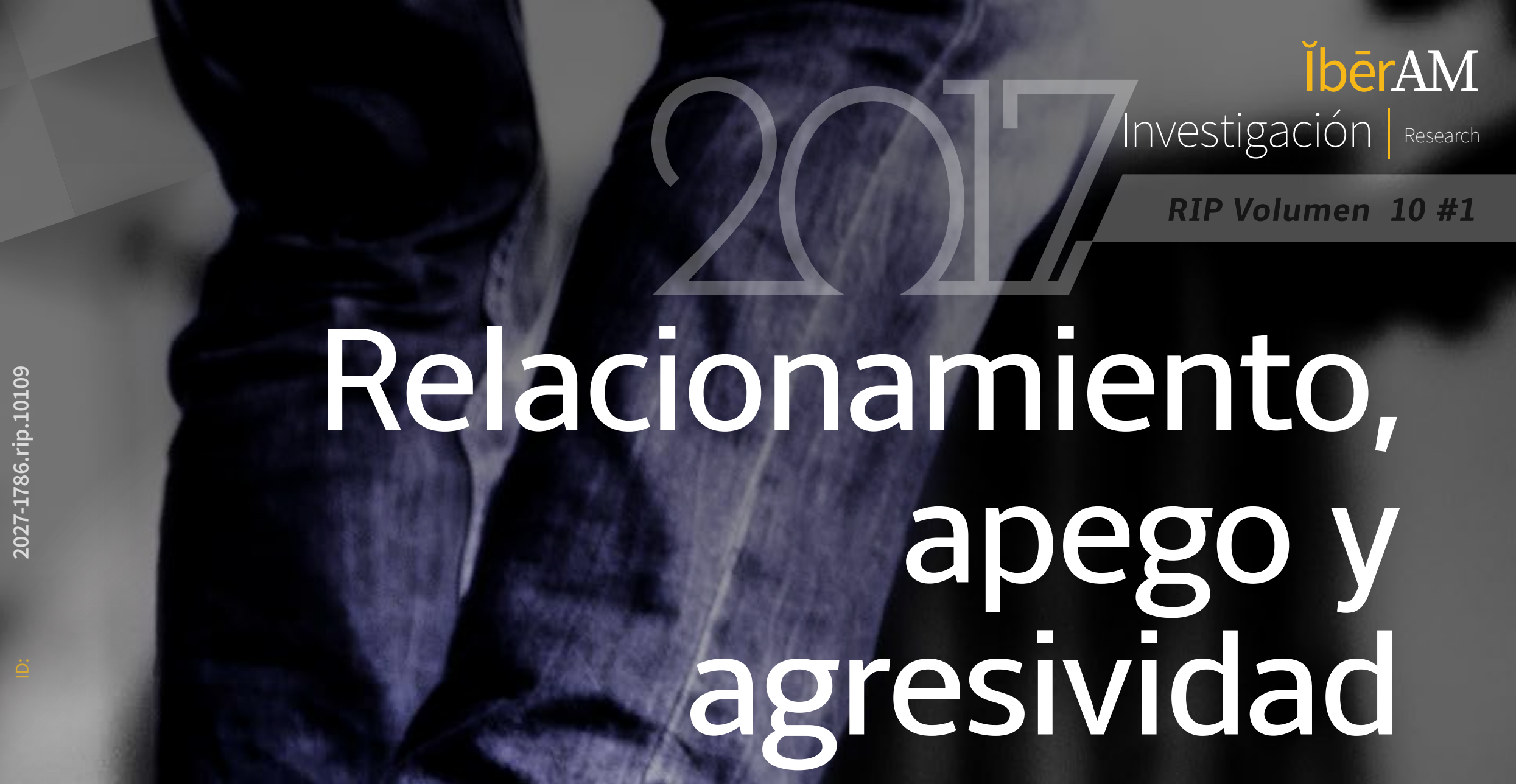

Preadolescentes de colegios públicos en Santa Marta, Colombia

Relationship, attachment and aggression:

wipreadolescents of public schools in Santa Marta, Colombia
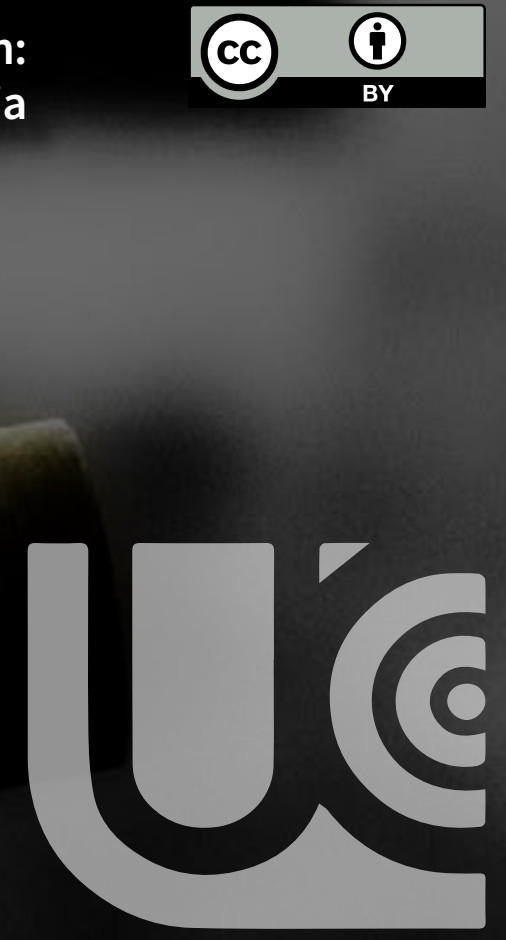

Revista Iberoamericana de

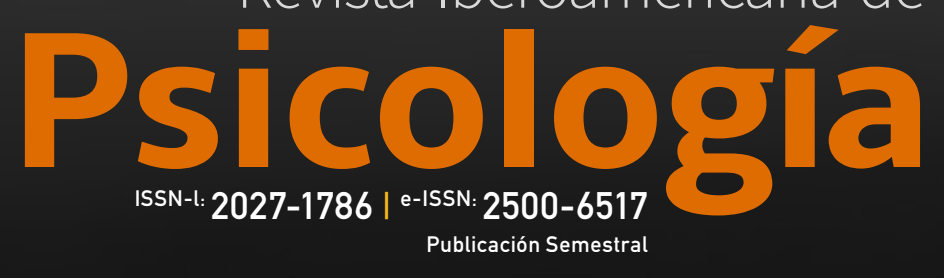


Revista Iberoamericana de

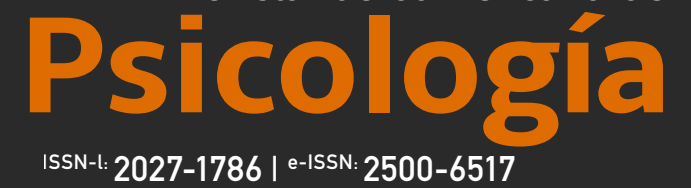

Publicación Semestral

ID:

2027-1786.rip.10109

Title: Relationship, attachment and aggression

Subtitle: Preadolescents of public schools in Santa Marta, Colombia

Título: Relacionamiento, apego y agresividad

Subtítulo: Preadolescentes de colegios públicos en Santa Marta, Colombia

Alt Title / Título alternativo:

[en]: Relating, attachment and aggressiveness in preadolescents of public schools of Santa Marta Colombia

[es]: Relacionamiento, apego y agresividad en preadolescentes de colegios públicos de Santa Marta Colombia

Author (s) / Autor (es):

\section{Alzate Duque, \& Zabarain Cogollo}

Keywords / Palabras Clave:

[en]

aggressiveness; attachment; alienation; communication; trust; bullying; peers

[es]: $\quad$ agresividad; apego; alienación; comunicación; confianza; intimidación escolar; pares

Financiación / Funding:

Colciencias, UCC Santa Marta

Submited: 2015-09-08

Acepted: 2017-06-26

\section{Resumen}

A través de esta caracterización de los tipos de relación entre preadolescentes con sus padres y compañeros, esta investigación pretende describir los elementos en la conexión con sus padres (en aspectos como el apego, la comunicación, la confianza, la alienación, la distancia familiar y el bloqueo

parental) e identifica elementos como

comportamiento agresivo o intimidación escolar (cuando está presente en la relación con sus compañeros).

La muestra se obtuvo en un grupo de 70 estudiantes al azar de 5 colegios públicos en Santa Marta, Colombia; estos cursaban sexto grado, entre 8 y 12 años, con un margen de error de 10\%. Los datos del estudio fueron

recolectados con herramientas como el

Test de Figuras Humanas (Koppitz, 1982),

la Prueba Familiar de Corman (1961), el

IPPA: Inventario de Apego con Padres y

Pares (Armsden \& Greenberg, 1987; Pardo,

Pineda, Carrillo y Castro, 2006), y la Escala

Abreviada del Cuestionario de Intimidación Escolar (Moratto, Cárdenas, \& Berbesí, 2012).

Los resultados obtenidos destacan una comunicación moderada-baja y la confianza

de los preadolescentes hacia sus padres,

con bajos índices de alienación y percepción

de distanciamiento familiar; Además, los

tipos de apego que predominan entre ellos son ansioso-inseguros, ambivalentes y evasivos. En cuanto a las relaciones con los compañeros, el índice inconsciente de agresión e impulsividad fueron pruebas, con bajos sentimientos de culpa, así como

la manifestación mínima consciente de

la admisión de algún tipo de intimidación escolar. Además, el estudio identifica que las prácticas más frecuentes de esta población implican signos de agresividad pasiva como exclusión, ocultamiento de cosas y ser llamado por sobrenombres.

\section{Abstract}

Across this characterization of the relationship types between preadolescents with their parents and peers, this research aims to describe the elements in the hookup with their parents (in aspects as the attachment, communication, trust, alienation, family distance and parental block) and identifies elements as aggressive behavior or school bullying (when is present in the relationship with their peers).

The sample was obtained in a 70 random students group of 5 public schools in Santa

Marta, Colombia; they were coursing 6th degree, between 8 to 12 years old, with an error margin of $10 \%$. The study data were collect with tools like the Koppitz Human Figure Test (1982), the Corman

Family Test (1961), the IPPA: Parent and Paired Attachment Inventory (Armsden \& Greenberg, 1987; Pardo, Pineda, Carrillo, \& Castro, 2006), and the Abbreviated Scale of the School Bullying Questionnaire (Moratto, Cárdenas, \& Berbesí, 2012). The obtained results remark a moderate-low communication and confidence from the preadolescents towards their parents, with

low indexes of alienation and perception

of family distancing; Also, the types of

attachment that predominate among

them are anxious-insecure, ambivalent and avoidant. Regarding relationships with peers, unconscious index of aggression and impulsivity were evidence, with low feelings

of guilt, as well as the minimal conscious manifestation of admission of some type of school intimidation. In addition, the study identifies that the most frequent practices of this population involve signs of passive aggressiveness as exclusion, hiding things, and being call by nicknames.
Viviana Marcela Alzate Duque, MA Psisp

ORCID: $\quad$ 0000-0002-5018-6101

Source I Filiacion:

Universidad Cooperativa de Colombia

BIO:

Psicologa - Magister en desarrollo integral en niños

y adolescentes - pos grado en actuación clínica psicoanalítica

City I Ciudad:

Santa Marta [co]

e-mail:

viviana.alzate@campusucc.edu.co
Dra Sara J. Zabarain Cogollo, MSc Psi sp

ORCID: 0000-003-1685-0715

Source I Filiacion:

Universidad Cooperativa de Colombia

BIO:

Psicologa-Especialista en Psicología Clínica, Magister en

Psicología, Doctor en Psicología

City I Ciudad:

Santa Marta [co]

e-mail:

sara.zabarain@campusucc.edu.co

Citar como:

Alzate Duque, V. M., \& Zabarain Cogollo, S. J. (2017). Relacionamiento, apego y agresividad: Preadolescentes de colegios públicos en Santa Marta, Colombia. Revista Iberoamericana de Psicología issn-l:2027-1786, 10 (1), 73-81.

Obtenido de: https://revistas.iberoamericana.edu.co/index.php/ripsicologia/article/view/811 


\title{
Relacionamiento, apego y agresividad Preadolescentes de colegios públicos en Santa Marta, Colombia
}

\author{
Relationship, attachment and aggression: \\ Preadolescents of public schools in Santa Marta, Colombia \\ Viviana Marcela Alzate Duque \\ Sara J. Zabarain Cogollo
}

\section{Introducción}

Los desarrollos en torno a la teoría del apego permitieron la constitución de uno de los campos más prolíficos de investigación interdisciplinaria en ciencias sociales ${ }^{1}$. Partiendo de estudios como los de Herbert Mead (1863 - 1931), los investigadores en Psicología del Desarrollo se interesaron por explorar (además del desarrollo cognitivo o motor, por ejemplo, temas de interés predominante en la psicología del desarrollo de principios del siglo XX), (Yáñez, 2012) la importancia de los vínculos afectivos en el desarrollo y, en especial, de las pautas de relacionamiento afectivo construidas entre padres e hijos. El concepto central sobre el cual se centrará la investigación en torno a este tema será el de apego (attachment).

\footnotetext{
1 Mead (1948, citada por LLoyds, 2001), combinando el campo de la antropología cultural y la investigación, estudió las pautas de crianza en diferentes tribus y describió relaciones entre las primeras experiencias de vida y la conducta adulta. Tras considerar que las prácticas de crianza de los padres no tenían efecto alguno en los hijos, Mead se interesa entonces en temas alrededor de la separación de hijos y madres, abriendo nuevas líneas de investigación. Sus hallazgos tras estudiar la tribu caníbal mundumumor y la tribu de los Arapesh son de especial importancia: En la tribu caníbal mundugumor las madres cargaban a los bebés en cestas duras y los alimentaban de pie, procuraban mantenerlos hambrientos para que mamaran vigorosamente y les retiraban el pecho en cuanto notaban que empezaban a saciarse. El carácter que desarrollaban estos infantes en la edad adulta era ávido y furioso, lo cual se manifestaba incluso en sus relaciones sexuales, las cuales incluían mordiscos y rasguños como si se tratase de un combate. Por su parte los Arapesh criaban a sus hijos con sumo cariño, ofreciéndoles y proveyéndoles todo, quienes en su adultez adoptaban una actitud pasiva y receptiva frente a los demás. Tras analizar estas dinámicas Mead sugirió que los bebés, cuando son alimentados, aprenden sobre la predisposición de los demás a atender y satisfacer sus necesidades o a ignorarlas y rehusarlas. Y es a partir de estas experiencias que eventualmente organizarán su visión del mundo y sus estilos de respuesta.
}

En términos generales la Teoría del Apego establece que el vínculo afectivo construido en la interacción entre padres e hijos en la infancia es determinante para la forma en que se organizan las pautas de interacción y relacionamiento con otros durante todo el resto de la vida. El apego, según plantea (Urizar, 2012), es un mecanismo pre-programado que activa toda una gama de comportamientos, posibilitando la vinculación bebé-madre bajo un fin biológico específico: proveer, por parte del cuidador, la proximidad, protección y seguridad que el infante necesita para emprender la exploración por un mundo que le resulta desconocido.

De acuerdo con la teoría del apego, el tipo de vínculo afectivo que se haya establecido con los padres en la interacción con los primeros objetos durante la infancia resulta determinante de los comportamientos posteriores presentes a lo largo del desarrollo. La comunicación establecida entre padres e hijos a través de sus vivencias influye en el modo en que se establecen las relaciones con otros (Garcés \& Palacio, 2010). El papel de la madre (o cuidador), en este proceso, resulta ser fundamental pues, como afirma Klein (2009), la constitución del vínculo afectivo construido con el hijo será determinante para la constitución de la identidad, el temperamento y el carácter, dependiendo de si satisface o no las necesidades del niño. La psicología del desarrollo ha mostrado, por ejemplo, si no existe confianza y seguridad en las figuras paternas, es poco probable que se confíe en otros. Así las cosas, cuando este 'objeto' fundamental que es la madre, es fuente de ambivalencia e inseguridad o cuando este se sienta sobre a base de la confianza y la seguridad, en ambos casos tendrán unos efectos directos sobre el desarrollo de la persona al nivel de su interacción con otros. El vínculo afectivo, en este sentido refiere al lazo afectivo que emerge entre dos personas (padres-e-hijos), y que determina el marco de confianza entre pares y con el contexto en general. 
Relacionamiento, apego y agresividad

Preadolescentes de colegios públicos en Santa Marta, Colombia

Los desarrollos de la Teoría del Apego llevados a cabo durante las décadas de los años 60 y 70 permitieron determinar unos patrones de apego 0 estilos de apego en los bebés, los cuales probaron guardar cierta relación con actitudes y eventos presentes en la edad adulta. Se evidenció que los niños que reflejaron un vínculo de apego seguro con sus padres fueron más propensos a ser socialmente competentes que sus pares; también se evidenciaron efectos al nivel de la sociabilidad, la regulación emocional, la evolución intelectual y la formación de la identidad social. En cambio, patrones de apego inestable en la infancia -de apego seguro a inseguro- tienden a correlacionar más con dificultades en el área social y la presencia de situaciones como enfermedades, abusos, muertes, divorcios u otras situaciones. Adicional a lo anterior, se determinó que aquellos niños que han sufrido de abuso o que han sido descuidados por sus padres, presentaron dificultades para desarrollar apegos seguros $y$, por el contrario, tendieron a presentar patrones de apego inseguro que persisten a lo largo de los años preescolares y parte de su vida adulta.

Así pues, las conductas vinculares determinan unos estilos de apego específicos que el niño construye a partir de la experiencia y proximidad con sus figuras cuidadoras, que se traducen en tipos de respuesta organizados a partir de la experiencia afectiva establecida en el vínculo madre (cuidador)-hijo. Estos estilos de apego fueron planteados por Bolwby (2006) en la década de los años 50 y fueron posteriormente ampliados por Ainsworth y Bell (1989). De acuerdo con Bowlby (2006), es posible identificar cuatro sistemas de conductas relacionados entre sí:

\ El sistema de conductas de apego: refiere a todas aquellas conductas que están al servicio del mantenimiento de la proximidad y contacto con las figuras de apego (sonrisas, llanto, contactos táctiles, etc.); estas conductas se activan cuando aumenta la distancia respecto a la figura de apego o cuando se perciben señales de amenazas, con el objetivo de restablecer la proximidad.

凶 El sistema de exploración: guarda estrecha relación y compatibilidad con el sistema anterior, en tanto la exploración del entorno disminuye cuando se activan las conductas de apego.

\ El sistema de miedo a los extraños: implica la disminución de las conductas exploratorias y el aumento de las conductas de apego.

囚 El sistema afiliativo: refiere al interés que muestran los individuos por mantener la proximidad e interactuar con otros, incluso con quienes no se han establecido vínculos afectivos, razón por la que guarda cierta incompatibilidad con el sistema de miedo a los extraños. Cabe agregar que éste sistema no es exclusivo de la especie humana.

Las investigaciones contemporáneas han mostrado que, por ejemplo, cuando se ha desarrollado un vínculo de apego seguro, el padre y la madre se constituyen como (Garrido-Rojas, 2006) la base afectiva para el establecimiento de la seguridad, la comunicación y la confianza que, a su vez, permiten el desarrollo de relaciones interpersonales adecuadas. Pero si, por el contrario, el niño o niña no recibe la contención afectiva necesaria -que fomenta también la tolerancia a la frustración y la regulación emocional- establecerá entonces relaciones ambivalentes, inseguras y, en muchos casos, cargadas de agresividad y desconfianza hacia los otros (Ainsworth \& Bell, 1989; Bión, 2000; Bowlby, 2006; Fonagy, 1999; Klein, 2009; Padilla, 2006; Rozenel, 2006; Varela \& Lecannelier, 2008; Winnicott, 1990). Estos vínculos débiles -inestables a través del tiempo, caracterizados por una comunicación y apoyo pobres, que no fomentan la confianza ni la generación de estrategias adecuadas frente al estrés- afectan a los individuos de modo que tienden a proyectaren los demás sus carencias afectivas. Así pues, estos individuos tienden a compensar estas carencias de afecto y cariño de parte de sus cuidadores adoptando una actitud de independencia y distanciamiento emocional (a pesar de tener lazos sociales), así como una actitud negativista. Otra forma de apego inadecuado es el apego desorganizado, caracterizado por la total ausencia de estrategias que ayudan a organizar una respuesta adecuada ante el incomodidad e inseguridad que produce una situación extraña y estresante. Investigaciones indican que la falta de un vínculo afectivo adecuado en la relación madre-hijo puede influir en problemas de conducta en la escuela primaria y secundaria, problemas con los pares, y problemas con los padres (Carlson, 1998; Lyons, Alpern , \& Repacholi, 1993; Lyons, 1996; Méndez \& González, 2002).

De acuerdo con lo anterior, estas formas o estilos de apego resultan ser fundamentales para el desarrollo posterior, pues determinan los modelos de relacionamiento a partir de los cuales se guiarán las interacciones de las personas con sus congéneres y con el mundo. (Oliva, 2004). La importancia fundamental de la teoría del apego radica entonces en comprender que los seres humanos, desde el nacimiento, necesitan desarrollar una relación con al menos un cuidador principal para que su desarrollo social y emocional sea lo más normal posible y se eviten modelos de relacionamiento patológicos. Los modelos de relacionamiento, entonces, se construirían en función de los tipos de apego establecidos. La investigación psicológica nos ha mostrado que una buena comunicación y poco distanciamiento familiar fomentan la seguridad, confianza y estima entre hijos y cuidadores/padres, lo cual provee al individuo una base segura que le permite relacionarse con sus cuidadores, pares y otros de manera sana y segura. Asimismo, sabemos que los modelos de relacionamiento basados en apegos inseguros, evitativos y ambivalentes, generan situaciones diádicas de comportamiento agresivo constante y permanente, así como de intimidación escolar (Cepeda, Pacheco, García , \& Piraquive, 2008; Olweus, 1998; Santamaría, 2013; Zurita, 2011; Zabarain \& Sánchez, 2009)²

A partir de todos estos postulados teóricos y evidencias de investigaciones anteriores surge esta investigación, la cual pretende conocer los modelos de relacionamiento de los preadolescentes y entenderlos como formas de expresión propias a partir de los tipos de apego establecidos e internalizados en su infancia (Fairbairn, 2002), y comprender el comportamiento agresivo y la intimidación escolar como productos de la alienación parental, el distanciamiento familiar (específicamente de los adolescentes respecto a sus padres), y la escasa confianza y comunicación entre padres e hijos.

\section{Metodología}

\section{Diseño}

El tipo de diseño empleado en este estudio fue descriptivo, en tanto su finalidad fue describir los modelos de relacionamiento (Apego, comunicación, confianza y alienación parental) así como el comportamiento agresivo (intimidación escolar) de los preadolescentes

\footnotetext{
2 En lo que respecta a la cultura latinoamericana, específicamente en países como Colombia, Chile, Costa Rica, Perú y Venezuela, se han realizado estudios que asocian los comportamientos agresivos con situaciones familiares disfuncionales (Valencia y Henao, 2012) así como con determinados tipos de apego establecidos durante la infancia. Entre sus hallazgos se evidenciaron tipos de apego inseguro - caracterizado por la ambivalencia afectiva o por la evitación completa de la figura de afecto-, elementos intra-psíquicos en preadolescentes, con pulsiones agresivas producto de su exposición, cuando niños, a situaciones de violencia activa, a su percepción de no-aceptación por parte de sus cuidadores (Varela \& Lecannelier, 2008; Berdugo y Amar, 2006;; Cubero Venegas, 2004) y a su identificación con su agresor (objetos primarios).
} 
que participaron en el estudio. Los estudios descriptivos, según, Hernández Sampieri, Fernández Collado y Baptista Lucio (1994), son aquellos que buscan recolectar datos y evidencia con el fin de describir un fenómeno específico de la realidad que puede o no haber sido explorado previamente.

\section{Participantes}

La población-universo objeto del presente estudio la conformaron $\mathbf{2 8 0}$ niños y niñas pertenecientes a cinco (5) colegios públicos de Santa Marta, con los cuales tiene convenio la facultad de psicología de la Universidad Cooperativa de Colombia. Los criterios de inclusión fueron los siguientes: que fuesen niños de ambos géneros (femenino y masculino) cuyas edades oscilaran entre los ocho (8) y doce (12) años; que estuviesen matriculados -en calidad de estudiantes activosy estuviesen cursando sexto grado $\left(6^{\circ}\right)$; no se tuvo en cuenta el tipo de familia, ni reportes relacionados con comportamiento agresivo. De esta población se seleccionó inicialmente una muestra, de manera aleatoria teniendo en cuenta un margen de error del 10\%, con lo que se seleccionaron un total de $\mathbf{7 2}$ niños y niñas. Posteriormente se retiraron dos (2) individuos del estudio, de manera voluntaria, para un total de $\mathbf{7 0}$ niños quienes conformaron la muestra final.

Es preciso mencionar que se tuvieron en cuenta todas las normas éticas de la Psicología, en especial las contempladas en el Código Ético de la Ley del Psicólogo en Colombia, Ley 1090 de 2006. Se contó con el consentimiento informado de los padres, así como el consentimiento institucional para poder llevar a cabo la investigación, y a la información recabada le dio un manejo confidencial en todo momento.

\section{Instrumentos}

Los instrumentos empleados para recabar datos y analizarlos posteriormente fueron los siguientes:

\section{El Test de la Figura Humana}

Prueba diseñada por Koppitz (1982), técnica de expresión de dibujo sencilla para los niños que permite identificar elementos inconscientes de la personalidad a través del análisis de los rasgos y trazos de los dibujos de hombre y mujer que se hacen de manera libre.

\section{Test de la Familia}

Posibilita que la persona a quien se le aplica proyecte estados emocionales y percepciones respecto su familia. Este test permite analizar aspectos como la dinámica familiar, la estructura familiar y los vínculos afectivos, en términos de Corman (1961)

\section{El Inventario de Apego con Padres y Pares (IPPA)}

Herramienta de Armsdem y Greenberg (1987), utilizado para medir la calidad del apego percibida por los adolescentes hacia sus padres, en su versión adaptada a la población colombiana de (Pardo, Pineda, Carrillo, \& Castro, 2006) para los fines de esta investigación. Este inventario está conformado por $\mathbf{7 5}$ afirmaciones divididas en tres categorías (mamá - papá - pares), cada incluyendo $\mathbf{2 5}$ preguntas. Es preciso anotar que para el presente estudio se aplicaron los ítems de padres en tanto el interés era medir la relación paterno-filial.

Esta escala presenta un Índice de Consistencia Interna total de $\mathbf{0 . 6 0}$, donde la consistencia interna para la escala de la madre es de 0.90 y para la escala del padre es de $\mathbf{0 . 9 3}$. Los ítems se califican con una Escala Likert de $\mathbf{5}$ puntos, donde 1 corresponde a casi nunca y 5 casi siempre o siempre. Este instrumento permite medir tres dimensiones: Confianza, Comunicación y Alienación. La primera mide el grado de comprensión y respeto mutuo en la relación; la comunicación mide la amplitud y calidad de la comunicación; y la alienación evalúa los sentimientos de ira y la no-involucración emocional en las relaciones interpersonales.

\section{El Cuestionario Breve de Intimidación Escolar}

-En una versión abreviada del CIE-A- que mide los índices de acoso escolar a partir de tres componentes: victimización, sintomatología y victimarios (Moratto, Cárdenas, \& Berbesí, 2012)

La aplicación de todos los instrumentos se realizó de manera individual, en un espacio adecuado en pro de la confidencialidad. Una vez recabados los datos, se empleó el software estadístico SPSS vs.18 para su análisis.

\section{Procedimiento}

Fase uno: Se invitó a participar de la investigación a las quince (15) I.E.D de la ciudad de Santa Marta que acogen población mixta, de las cuales cinco (5) aceptaron participar.

Fase dos: Se llevó a cabo una socialización a docentes, coordinadores, rectores y padres de familia sobre los objetivos de la investigación y se realizó la firma de los consentimientos informados.

Fase tres: Aplicación de los instrumentos para recabar datos, los cuales fueron posteriormente tabulados y analizados empleando el software estadístico SPSS vs.18. Se utilizaron las frecuencias y porcentajes de los estadísticos descriptivos del software.

Fase cuatro: Divulgación/socialización de los resultados de la investigación en los colegios; presentación del artículo producto de dicha investigación y presentación del mismo como ponencia internacional.

\section{Resultados}

El análisis de los modelos de relacionamiento de los preadolescentes que conformaron la muestra del presente estudio se realizó a partir de los resultados de la Prueba IPPA (Pardo, Pineda, Carrillo, \& Castro, 2006), teniendo en cuenta tres aspectos del relacionamiento con la madre y el padre: tipo de apego, confianza, comunicación y alienación.

Se muestran a continuación los resultados más significativos de los tipos de apego con el padre y la madre. 


\section{Relacionamiento, apego y agresividad}

Preadolescentes de colegios públicos en Santa Marta, Colombia

Tabla 1 Distribución de los tipos de apego con el padre y la madre

\begin{tabular}{ccc}
\hline Estilos de Apego & Padre & Madre \\
\hline Ambivalentes & 37,2 & 67,3 \\
\hline Evitativo & 45,2 & 18,7 \\
Seguro & 17,6 & 14,0 \\
\hline
\end{tabular}

Fuente: elaboración propia

Se evidencia una diferencia cuantitativa en cada tipo de apego respecto a la madre y el padre.

Los resultados en la tabla 1 indican que el $\mathbf{4 5 . 2 \%}$ de los participantes presenta un tipo de apego evitativo con el padre, y el $\mathbf{6 7 . 3} \%$ presenta un tipo de apego ambivalente con la madre. Existe una mayor tendencia en la población hacia los apegos inseguros con el padre, pero se percibe mayor ambivalencia en la relación afectiva con la madre.

A continuación, se observan los resultados sobre la confianza en la madre y el padre.

Tabla 2 Indicadores de confianza en la madre y el padre

\begin{tabular}{|c|c|c|c|c|c|}
\hline Pregunta Indicadores maternos & CNC & NFC & AVC & CFC & $\csc$ \\
\hline Respeto por los sentimientos & $15 \%$ & $23 \%$ & $8 \%$ & $15 \%$ & $39 \%$ \\
\hline Trabajo como padre o madre & $0 \%$ & $15 \%$ & $8 \%$ & $31 \%$ & $46 \%$ \\
\hline Aceptación de los padres & $0 \%$ & $15 \%$ & $0 \%$ & $31 \%$ & $54 \%$ \\
\hline Aceptación del punto de vista & $16 \%$ & $23 \%$ & $15 \%$ & $15 \%$ & $31 \%$ \\
\hline Confianza en el juicio del evaluado & $23 \%$ & $0 \%$ & $8 \%$ & $23 \%$ & $46 \%$ \\
\hline $\begin{array}{r}\text { Percepción de entendimiento de parte de los } \\
\text { padres }\end{array}$ & $8 \%$ & $0 \%$ & $8 \%$ & $38 \%$ & $46 \%$ \\
\hline Comprensión de los padres ante la rabia & $15 \%$ & $8 \%$ & $0 \%$ & $23 \%$ & $54 \%$ \\
\hline Confianza en los padres & $8 \%$ & $8 \%$ & $0 \%$ & $23 \%$ & $61 \%$ \\
\hline Pregunta Indicadores paternos & CNC & NFC & AVC & CFC & $\csc$ \\
\hline Respeto por los sentimientos & $8 \%$ & $0 \%$ & $15 \%$ & $31 \%$ & $46 \%$ \\
\hline Trabajo como padre o madre & $0 \%$ & $0 \%$ & $30 \%$ & $10 \%$ & $60 \%$ \\
\hline Aceptación de los padres & $15 \%$ & $8 \%$ & $8 \%$ & $23 \%$ & $46 \%$ \\
\hline Aceptación del punto de vista & $23 \%$ & $8 \%$ & $8 \%$ & $23 \%$ & $38 \%$ \\
\hline Confianza en el juicio del evaluado & $23 \%$ & $15 \%$ & $0 \%$ & $23 \%$ & $39 \%$ \\
\hline $\begin{array}{r}\text { Percepción de entendimiento de parte de los } \\
\text { padres }\end{array}$ & $15 \%$ & $8 \%$ & $0 \%$ & $23 \%$ & $54 \%$ \\
\hline Comprensión de los padres ante la rabia & $7 \%$ & $8 \%$ & $8 \%$ & $31 \%$ & $46 \%$ \\
\hline Confianza en los padres & $15 \%$ & $0 \%$ & $8 \%$ & $23 \%$ & $54 \%$ \\
\hline
\end{tabular}

CNC=Casi nunca es cierto; $\mathbf{N F C = N o}$ muy frecuentemente es cierto; AVC=Algunas veces es cierto; $\mathbf{C F C}=$ Con frecuencia es cierto; $\mathbf{C S C}=$ Casi siempre o siempre es cierto | Porcentajes a nivel de indicadores que miden la confianza que tienen los preadolescentes en su madre y padre; Fuente: elaboración propia

Los resultados indican que sólo el $\mathbf{3 9} \%$ de los preadolescentes consideran que su madre respeta sus sentimientos; el $\mathbf{4 6} \%$ piensa que su madre hace un buen trabajo como mamá y solo el $\mathbf{3 1} \%$ piensa que su madre tiene en cuenta su punto de vista. Por el contrario, se puede observar que el $\mathbf{4 6} \%$ de los preadolescentes considera que su padre respeta sus sentimientos; el $\mathbf{6 0} \%$ considera que su padre hace un buen trabajo como papá y el $\mathbf{3 8} \%$ considera que su padre tiene en cuenta su punto de vista.

Estos resultados indican que sólo una partede los preadolescentes que participaron en esta investigación perciben a su madre como figura protectora en caso de requerir ayuda u orientación. Al realizar la comparación entre la confianza hacia la madre y hacia el padre, podemos observar que existe una tendencia hacia la confianza en la madre, aunque la diferencia entre estos dos cuidadores no es significativa. Los preadolescentes participantes consideran que su madre respeta su punto de vista más que el padre. Los puntajes más bajos refieren a la percepción de poca confianza de parte de sus padres, lo que puede influir en su propio juicio frente a situaciones de la vida cotidiana, y generar dependencia afectiva o debilidad en la toma de decisiones. La confianza hace referencia al desarrollo de hábitos en los padres orientados a generar en sus hijos habilidades de escucha, diálogo y criterio (Oliva, 2006).

A continuación, se muestran los resultados referentes a la comunicación con la madre y el padre.

Tabla 3 Indicadores de comunicación con la madre y el padre

\begin{tabular}{|c|c|c|c|c|c|}
\hline Pregunta | Indicadores maternos & CNC & NFC & AVC & CFC & CSC \\
\hline $\begin{array}{l}\text { Me gusta saber la opinión de mi mamá } \\
\text { cuando hay cosas que me preocupan }\end{array}$ & $0 \%$ & $15 \%$ & $8 \%$ & $31 \%$ & $46 \%$ \\
\hline Mi mamá sabe cuándo estoy molesto por algo & $0 \%$ & $15 \%$ & $0 \%$ & $39 \%$ & $46 \%$ \\
\hline $\begin{array}{r}\text { Mi mamá me ayuda a entenderme mejor a mí } \\
\text { mismo }\end{array}$ & $0 \%$ & $0 \%$ & $15 \%$ & $39 \%$ & $46 \%$ \\
\hline $\begin{array}{r}\text { Le cuento a mi mamá sobre mis problemas y } \\
\text { dificultades }\end{array}$ & $7 \%$ & $8 \%$ & $0 \%$ & $31 \%$ & $54 \%$ \\
\hline $\begin{array}{r}\text { Mi mamá me ayuda a hablar sobre mis } \\
\text { dificultades }\end{array}$ & $23 \%$ & $15 \%$ & $0 \%$ & $23 \%$ & $39 \%$ \\
\hline $\begin{array}{r}\text { Puedo contar con mi mamá cuando necesito } \\
\text { desahogarme }\end{array}$ & $0 \%$ & $7 \%$ & $8 \%$ & $31 \%$ & $54 \%$ \\
\hline $\begin{array}{l}\text { Si mi mamá sabe que algo me está } \\
\text { molestando, me pregunta sobre el tema }\end{array}$ & $8 \%$ & $0 \%$ & $15 \%$ & $23 \%$ & $54 \%$ \\
\hline Pregunta | Indicadores paternos & CNC & NFC & AVC & CFC & csc \\
\hline $\begin{array}{r}\text { Me gusta saber la opinión de mi papá cuando } \\
\text { hay cosas que me preocupan }\end{array}$ & $29 \%$ & $0 \%$ & $14 \%$ & $43 \%$ & $14 \%$ \\
\hline Mi papá sabe cuándo estoy molesto por algo & $16 \%$ & $8 \%$ & $15 \%$ & $15 \%$ & $46 \%$ \\
\hline $\begin{array}{r}\text { Mi papá me ayuda a entenderme mejor a mí } \\
\text { mismo }\end{array}$ & $8 \%$ & $0 \%$ & $15 \%$ & $23 \%$ & $54 \%$ \\
\hline $\begin{array}{r}\text { Le cuento a mi papá sobre mis problemas y } \\
\text { dificultades }\end{array}$ & $31 \%$ & $15 \%$ & $0 \%$ & $23 \%$ & $31 \%$ \\
\hline $\begin{array}{r}\text { Mi papá me ayuda a hablar sobre mis } \\
\text { dificultades }\end{array}$ & $15 \%$ & $8 \%$ & $0 \%$ & $23 \%$ & $54 \%$ \\
\hline $\begin{array}{r}\text { Puedo contar con mi papá cuando necesito } \\
\text { desahogarme }\end{array}$ & $15 \%$ & $8 \%$ & $23 \%$ & $23 \%$ & $31 \%$ \\
\hline $\begin{array}{l}\text { Si mi papá sabe que algo me está } \\
\text { molestando, me pregunta sobre el tema }\end{array}$ & $23 \%$ & $0 \%$ & $15 \%$ & $23 \%$ & $39 \%$ \\
\hline
\end{tabular}

CNC=Casi nunca es cierto; NFC=No muy frecuentemente es cierto; $\mathbf{A V C}=$ Algunas veces es cierto; $\mathbf{C F C}=$ Con frecuencia es cierto; $\mathbf{C S C}=$ Casi siempre o siempre es cierto | Se evidencia los niveles de comunicación existente entre la madre y/o padre y los preadolescentes; Fuente: elaboración propia

Los resultados indican que el $\mathbf{5 4} \%$ de los preadolescentes cuenta a su mamá sobre sus problemas; el $\mathbf{5 4 \%}$, considera que cuenta con su madre cuando necesita desahogarse y el $\mathbf{5 4 \%}$ considera que su madre le pregunta cuando algo le está molestando. Respecto a la comunicación con el padre, el $\mathbf{5 4} \%$ de los preadolescentes piensa que su papá le ayuda a entenderse a sí mismo y siente que le ayuda a hablar sobre sus dificultades. De igual forma el $\mathbf{4 6} \%$ de los preadolescentes de esta investigación piensa que su papá sabe cuándo está molesto por algo.

Los preadolescentes participantes perciben más a la madre como figura con la cual se pueden desahogar, sin embargo, hay bajos promedios respecto a la percepción de que la madre realmente les ayude. En cuanto a lo que al padre refiere, lo perciben más como una figura orientadora con la que pueden hablar, a pesar de que prefieran no saber la opinión de éste cuando ciertas cosas les preocupan y que sientan que no pueden contar con él cuando necesitan desahogarse.

A continuación, se muestran los resultados de alienación parental hacia la madre y el padre. 
Tabla 4 Indicadores de alienación hacia la madre y el padre

\begin{tabular}{|c|c|c|c|c|c|}
\hline Pregunta | Variables maternas & CNC & NFC & AVC & CFC & $\csc$ \\
\hline $\begin{array}{l}\text { Me siento avergonzado y tonto cuando hablo } \\
\text { con mi mamá sobre mis problemas. }\end{array}$ & $54 \%$ & $15 \%$ & $8 \%$ & $0 \%$ & $23 \%$ \\
\hline $\begin{array}{l}\text { Cuando estoy con mi mamá me enojo } \\
\text { fácilmente }\end{array}$ & $17 \%$ & $17 \%$ & $8 \%$ & $25 \%$ & $33 \%$ \\
\hline $\begin{array}{l}\text { Me siento mal en muchas más ocasiones de } \\
\text { las que mi mamá se entera }\end{array}$ & $54 \%$ & $15 \%$ & $0 \%$ & $8 \%$ & $23 \%$ \\
\hline Siento rabia hacia mi mamá & $69 \%$ & $16 \%$ & $0 \%$ & $0 \%$ & $15 \%$ \\
\hline Mi mamá no me presta mucha atención & $54 \%$ & $8 \%$ & $15 \%$ & $8 \%$ & $15 \%$ \\
\hline $\begin{array}{l}\text { Mi mamá no entiende por lo que estoy } \\
\text { pasando en este momento }\end{array}$ & $31 \%$ & $8 \%$ & $8 \%$ & $15 \%$ & $38 \%$ \\
\hline Pregunta | Variables paternas & CNC & NFC & AVC & CFC & $\csc$ \\
\hline $\begin{array}{l}\text { Me siento avergonzado y tonto cuando hablo } \\
\text { con mi papá sobre mis problemas. }\end{array}$ & $62 \%$ & $0 \%$ & $0 \%$ & $15 \%$ & $23 \%$ \\
\hline $\begin{array}{l}\text { Cuando estoy con mi papá me enojo } \\
\text { fácilmente }\end{array}$ & $69 \%$ & $8 \%$ & $0 \%$ & $8 \%$ & $15 \%$ \\
\hline $\begin{array}{l}\text { Me siento mal en muchas más ocasiones de } \\
\text { las que mi papá se entera }\end{array}$ & $46 \%$ & $16 \%$ & $8 \%$ & $15 \%$ & $15 \%$ \\
\hline Siento rabia hacia mi papá & $69 \%$ & $16 \%$ & $0 \%$ & $0 \%$ & $15 \%$ \\
\hline Mi papá no me presta mucha atención & $46 \%$ & $7 \%$ & $8 \%$ & $8 \%$ & $31 \%$ \\
\hline $\begin{array}{l}\text { Mi papá no entiende por lo que estoy } \\
\text { pasando en este momento }\end{array}$ & $31 \%$ & $23 \%$ & $16 \%$ & $15 \%$ & $15 \%$ \\
\hline
\end{tabular}

CNC=Casi nunca es cierto; $\mathbf{N F C = N o ~ m u y ~ f r e c u e n t e m e n t e ~ e s ~ c i e r t o ; ~}$ $\mathbf{A V C}=$ Algunas veces es cierto; $\mathbf{C F C}=$ Con frecuencia es cierto; $\mathbf{C S C}=$ Casi siempre o siempre es cierto | Se correlaciona los niveles de alineación con la madre frente al padre; Fuente: Elaboración propia

De acuerdo con esto no se observan porcentajes altos relacionados a la dependencia o apego a la madre. Contrario a esto, el $\mathbf{3 8 \%}$ de los preadolescentes piensa que su madre no entiende lo que siente. Respecto a la alienación hacia la figura paterna, el $\mathbf{2 3} \%$ de los preadolescentes se siente avergonzado cuando habla con su papá sobre sus problemas y el $\mathbf{3 1} \mathbf{2}$ de ellos siente que su padre no le presta atención. A la población de preadolescentes de esta investigación no parece interesarle una relación cercana con su padre ni parece sentirse afectada por lo que él piense.

Adicional a lo anterior, se tuvo en cuenta el Test de Familia para observar la percepción que tienen los preadolescentes sobre el distanciamiento familiar y sobre su bloque parental.

A continuación, los resultados de la percepción de los preadolescentes sobre el distanciamiento familiar.

Tabla 5 Percepción del distanciamiento familiar

\begin{tabular}{|ccc|}
\hline Indicadores & Presente & No presente \\
\hline Cercanía familiar & $23 \%$ & $77 \%$ \\
\hline Distanciamiento familiar & $77 \%$ & $23 \%$ \\
\hline
\end{tabular}

Cada indicador se puntúa como $1=$ presente $00=$ No presente, siendo solo dos opciones de respuesta. Fuente: Elaboración propia

En lo que a la unión familiar refiere, el $\mathbf{7 7 \%}$ de los preadolescentes participantes de esta investigación percibe distanciamiento familiar, una falta de cercanía en la comunicación y en el compartir en la cotidianidad. parental.

A continuación, los resultados de la percepción del bloque Tabla 6 Percepción del bloque parental

\begin{tabular}{ccc}
\hline Indicadores & Presente & No presente \\
\hline Bloque parental Unido & $24 \%$ & $76 \%$ \\
\hline Percepción de distanciamiento entre los padres & $36 \%$ & $64 \%$ \\
No vinculación afectiva con los hermanos & $45 \%$ & $55 \%$ \\
No se incluye a sí mismo como parte de la familia & $22 \%$ & $78 \%$
\end{tabular}

Cada indicador se puntúa como 1=presente o 0=No presente, siendo sólo dos las opciones de respuesta; Fuente: elaboración propia
Respecto a los vínculos afectivos entre los miembros de la familia, sólo el $\mathbf{2 4 \%}$ de los preadolescentes percibe su bloque parental como unido. El $\mathbf{6 4 \%}$ percibe distanciamiento entre sus padres y el $\mathbf{5 5} \%$ no se siente vinculado afectivamente con sus hermanos, de hecho, parece haber cierta rivalidad entre ellos. Tan sólo el $\mathbf{2 2} \%$ de los sujetos no se consideran sí mismos como parte de su familia, pero a pesar de esto desean pertenecer a un grupo familiar. Es evidente que la unión y comunicación entre los miembros de su familia no es común.

En cuanto al comportamiento agresivo o intimidación se hallaron los siguientes resultados con base en el test de familia.

Tabla 7 Exteriorización del comportamiento agresivo: Agresividad hacia los demás

\begin{tabular}{ccc}
\hline Indicadores & Presente & No presente \\
\hline Agresividad y pobre control de impulsos & $54 \%$ & $46 \%$ \\
\hline Evasión y culpa & $46 \%$ & $54 \%$ \\
\hline
\end{tabular}

Cada indicador se puntúa como $1=$ presente 0 0=No presente; Fuente: elaboración propia

Respecto a la agresividad como manera de interacción, el $\mathbf{5 4 \%}$ de los preadolescentes presenta agresividad y pobre control de impulsos, sin sentimiento de culpa. Las interacciones sociales se dan con agresividad a manera de manifestación de su comunicación diaria.

A continuación, los resultados de intimidación escolar teniendo en cuenta el CIE-A.

Tabla 8 Situaciones de victimización por intimidación escolar

\begin{tabular}{|c|c|c|c|}
\hline Preguntas & Nunca & $\begin{array}{l}\text { Pocas } \\
\text { veces }\end{array}$ & $\begin{array}{c}\text { Muchas } \\
\text { veces }\end{array}$ \\
\hline No me dejan participar, me excluyen & $58 \%$ & $16 \%$ & $26 \%$ \\
\hline Me obligan a hacer cosas peligrosas para mi & $92 \%$ & $2 \%$ & $6 \%$ \\
\hline Rompen mis cosas a propósito & $88 \%$ & $6 \%$ & $6 \%$ \\
\hline Me esconden las cosas & $64 \%$ & $23 \%$ & $13 \%$ \\
\hline $\begin{array}{r}\text { Dicen a otros que no estén conmigo } \\
\text { o que no me hablen }\end{array}$ & $74 \%$ & $17 \%$ & $9 \%$ \\
\hline Me insultan & $78 \%$ & $14 \%$ & $8 \%$ \\
\hline Me pegan coscorrones, puñetazos, patadas & $91 \%$ & $6 \%$ & $3 \%$ \\
\hline Me chiflan o gritan & $78 \%$ & $13 \%$ & $9 \%$ \\
\hline Me desprecian & $92 \%$ & $5 \%$ & $3 \%$ \\
\hline Me llaman por apodos & $77 \%$ & $6 \%$ & $17 \%$ \\
\hline Me amenazan para que haga cosas que no quiero & $88 \%$ & $6 \%$ & $6 \%$ \\
\hline \multirow[t]{2}{*}{ Me obligan a hacer cosas que están mal } & $95 \%$ & $3 \%$ & $2 \%$ \\
\hline & \multicolumn{3}{|c|}{ Fuente: elaboración propi } \\
\hline
\end{tabular}

Respecto a lassituaciones devictimización, las más predominantes presentan un porcentaje muy bajo. El $\mathbf{2 6} \%$ de los preadolescentes considera que no lo dejan participar o lo excluyen, el $\mathbf{1 7 \%}$ manifiesta que lo llaman por apodos, y el $\mathbf{1 3} \mathbf{3}$ piensa que le esconden las cosas. Los resultados de la tabla 8 no evidencian situaciones de maltrato hacia otros. (Brendtro, 2001)

\section{Discusión}

El apego ambivalente que prima en la relación de los preadolescentes con su madre ocasiona sentimientos de ira o rabia inconscientemente encubiertos y cuya expresión no es clara, pero que se reflejan en una comunicación pobre y la poca confianza. Lo anterior guarda relación con la percepción de que tienen estos preadolescentes en consideran que su punto de vista no es aceptado, que no se les colabora ni se favorece una comunicación espontánea para hablar sobre sus dificultades. Por su parte, el apego evitativo con la figura paterna se manifiesta en la poca confianza que los preadolescentes tienen en los juicios que el padre emite. 


\section{Relacionamiento, apego y agresividad}

\section{Preadolescentes de colegios públicos en Santa Marta, Colombia}

Para que la comunicación familiar sea adecuada, sana y funcional, según (Antolínez, 1991) ésta debe ser: Clara, congruente, oportuna, receptiva, que explore la situación, se verifique y que tenga retroalimentación1, aspectos que no se manifiestan entre los sujetos de la investigación y sus padres.

En la adolescencia la figura de los padres adquiere otro significado, puede haber dificultades en la relación de los adolescentes con la autoridad, puede aminorar la confianza y la comunicación con sus cuidadores y así una tendencia a guardar secretos entre los grupos de amigos. Los adolescentes del presente estudio asumen una postura ambivalente, pues se observa que existe el deseo por conocer las opiniones de sus padres y comunicarse más afectivamente, a pesar de la percepción inconsciente de distanciamiento familiar en las interacciones entre los miembros de la familia y de percibirse sí mismos como inseguros frente al ambiente. Todas estas características indican que destaca en ellos un apego inseguro, ya que las bases del apego seguro son la confianza y la seguridad. (Ainsworth \& Bell, 1989; Spitz, 1994).

En cuanto a las relaciones con sus pares, tal como mencionó (Rozenel, 2006)), las bases afectivas de inseguridad y apegos inseguros generan contactos sociales ambivalentes. En el caso de la población objeto de estudio, no se observan manifestaciones conscientes de comportamiento agresivo, a pesar que sí existe en ellos agresividad a nivel inconsciente que tal vez no exteriorizan en las interacciones con sus pares. Sin embargo, la formación reactiva de estos sentimientos podría ocasionar dificultades a mediano y largo plazo en sus relaciones afectivas.

La relación entre el apego inseguro y ambivalente y la manifestación consciente de comportamientos agresivos hacia sus pares no se percibe con claridad en los actos externos de los adolescentes. Pero si existe en ellos agresividad reprimida y pobre control de impulsos que parten del apego inseguro y ambivalente, en tanto manifiestan sentir que su madre les causa enojo con facilidad y que no se sienten comprendidos por ella; la alienación parental es manifiesta en el discurso inconsciente de los adolescentes.

Se podría inferir que los sentimientos de enojo y desconfianza se transforman en otros contrarios como mecanismo defensivo, y se establecen miedos e inseguridades, pero luego se transforman en hostilidad sin sentimiento de culpa, propio de los apegos inseguros evitativos o rechazantes.

Por otra parte, el deseo de ser aceptado por los pares y de no ser excluido en la interacción social podrían llevar a que no se admita ser victimizado, lo que se puede manifestar en la relación con los pares en forma de alienación, tal como ocurre en los casos de apego inseguro ambivalente, en los que la inseguridad se mezcla con el deseo de confiar en el otro (Cerezo, 2006; Collell \& Escudé, 2002; Padilla, 2006).

La agresividad, o aceptación de la agresividad, puede ser un comportamiento asociado a las bases de supervivencia afectiva, producto de relaciones de apego poco seguras en las que no se perciben los objetos o padres como base de contención afectiva y seguridad. (Brendtro, 2001; Gagné, 2004; Gerd, Albrektsen, \& Qvarnstrøm, 2001; Rozenel, 2006).

\section{Conclusiones}

Para los preadolescentes no parece ser esencial la comunicación con sus padres, ni la confianza. La comunicación parece ser básica en torno a las preguntas hechas de forma directa, debido a que no perciben aceptación real de la expresión de sus sentimientos e ideas; incluso existe en ellos sentimientos de enojo, pero con signos bajos de alienación parental.

Priman en la muestra tipos de apegos inseguros y ambivalentes.

Los resultados de la investigación no evidencian signos de victimización o 'Bullying' quesean resultado de un acoso constanteyse identificó poca manifestación consciente de actos externos agresivos, pero existen de manera inconsciente agresividad e impulsividad con bajos sentimientos de culpa y hay frecuencia de prácticas de exclusión, esconder cosas y llamarse por apodos, todos signos inconscientes de agresividad pasiva.

\section{Referencias}

Ainsworth, M., \& Bell, S. (1989). Apego, exploración y separación, ilustrados a través de la conducta de niños de un año en una situación extraña. Madrid: Alianza.

Antolínez, B. (Julio - Diciembre de 1991). Comunicación Familiar. Avances en enfermeria, 9(2), 37-48. DOl: https://doi.org/10.15446/av.enferm

Armsden, G., \& Greenberg, M. (1987). The inventory of parent and peer attachment: Individual differences and their relationship to psychological well-being in adolescence. Journal of Youth and Adolescence, 16(5), $427-$ 454. DOI: https://doi.org/10.1007/BF02202939

Bión, W. (2000). Elementos de psicoanálisi. Buenos Aires, Argentina: Horme Paidos.

Bowlby, J. (2006). Vínculos afectivos: Formación, desarrollo y pérdida. Madrid: Morata.

Brendtro, L. (2001). Worse Than Sticks and stones: Lesson from research on ridicule. Reclaiming children and Youth, 47-49. Obtenido de: https:// search.proquest.com/openview/d6352d215cb2fbe66f5fb1c06f2cbab6/1? pq-origsite $=$ gscholar

Carlson, E. (1998). A prospective longitudinal study of attachment disorganization/ disorientation. Pubmed, 69(4), 11-28. Obtenido de https://www.ncbi.nlm. nih.gov/pubmed/9768489

Cepeda , E., Pacheco, P., García , L., \& Piraquive, C. (Septiembre de 2008). Acoso Escolar a Estudiantes de Educación Básica y Media. Revista de Salud Pública, 10(4), 517-528. Obtenido de: http://www.redalyc.org/articulo. oa?id $=42210402$

Cerezo, F. (2006). Violencia y victimización entre escolares: El bullying estrategias de identificación y elementos para la intervención. Revista Electronica de Investigacion Psicoeducaticon, 4(2), 333-352. Obtenido de: http:// www.sinohacesnadasosparte.org/Download/spanish/02 BULLYING_EN ESPANOL.pdf

Collell, J., \& Escudé, C. (Febrero de 2002). La violència entre iguals a l'escola: el Bullying. Àmbits de Psicopedagogia, 20-24. Obtenido de: http://enxarxats. intersindical.org/efemerides/violenentreiguals.pdf

Corman, L. (1961). Test del dibujo de la familia. Buenos Aires, Argentina: Kapelusz. Obtenido de: https://personalidadpreescolar.files.wordpress.com/2016/06/ test-de-la-familia-de-corman.pdf

Cubero Venegas, C. M. (2004). La disciplina en el aula: reflexiones en torno a los procesos de comunicación. Actualidades Investigativas en Educación, 4(2), 1-39. Obtenido de: http://www.redalyc.org/pdf/447/44740202.pdf

Fairbairn, W. (2002). Fairbairn and relational theory. New York, Estado Unidos: books Itda.

Fonagy, P. (13 de Mayo de 1999). Persistencias transgeneracionales del apego: una nueva teoría. Grupo psicoanalítico de discusión sobre el desarrollo. Obtenido de: http://www.aperturas.org/articulos. php?id=0000086\&a=Persistencias-ransgeneracionales-del-apego-unanueva-teoria 
Gagné. (2004). The socio- afecctive and Academic Impact of Early Entrance to School . Roeper Review, 26, 128-139.

Garcés, M., \& Palacio, J. (Enero - Junio de 2010). La comunicación familiar en asentamientos subnormales de Montería. Psicología desde el Caribe, 1-29. Obtenido de: http://www.redalyc.org/articulo.oa?id=21315106002

Gerd, K., Albrektsen, G., \& Qvarnstrøm, U. (2001). School- related stress experience as a risk factor for bullying behaviour. Journal of Youth and adolescence, 30(5), 561-575. Obtenido de: https://link.springer.com/ article/10.1023\%2FA\%3A1010448604838

Hernández Sampieri, R., Fernández Collado, C., \& Baptista Lucio, P. (1994). Metodología de la Investigación (600 ed.). México D.F. [mx]: McGraw-Hill.

Klein, M. (2009). Obras completas. Envidia y gratitud y otros trabajos. Buenos Aires, Argentina: Paidós.

Koppitz, E. (1982). El dibujo de la figura humana en los niños. Buenos Aires, Argentina: Guadalupe.

Ley 1090 de 2006, Por la cual se reglamenta el ejercicio de la profesión de Psicología, se dicta el Código Deontológico y Bioético y otras disposiciones (Congreso de la Republica de Colombia 6 de Septiembre de 2006). Obtenido de: http://colpsic.org.co/aym image/files/LEY 1090 DE 2006.pdf

Lyons, K. (1996). Attachment relationships among children with aggressive behavior problems: The role of disorganized early attachment patterns. Journal of Consulting and Clinical Psychology, 64(1), 64-73. Obtenido de: http://citeseerx.ist.psu.edu/viewdoc/ download?doi=10.1.1.463.4585\&rep=rep1\&type $=p d f$

Lyons, K., Alpern , L., \& Repacholi, B. (1993). Disorganized infant attachment classification and maternal psychosocial problems as predictors of hostile-aggressive behavior in the preschool classroom. Child development, 64(2), 572-585. Obtenido de: https://www.ncbi.nlm.nih.gov/ pubmed/8477635

Méndez, L., \& González, L. (2002). Descripción de patrones de apego en menores institucionalizados con problemas conductuales. Revista de Psicología de la Universidad de Chile(11), 75-92. Obtenido de: http://www. revistapsicologia.uchile.cl/index.php/RDP/article/viewFile/17288/18030

Moratto , N., Cárdenas, N., \& Berbesí, D. (Julio - Diciembre de 2012). Validación de un cuestionario breve para detectar intimidacion escolar. Revista CES Psicología, 5(2), 70-78. Obtenido de: http://www.scielo.org.co/pdf/cesp/ v5n2/v5n2a06.pdf

Oliva , A. (2004). Estado Actual de la Teoría del Apego. Revista de Psiquiatría y Psicología del Niño y del Adolescente. Universidad de Sevilla, 4(1), 65-81. Obtenido de: $h$ ttp://chitita.uta.cl/cursos/2012-1/0000636/recursos/r-9.pdf
Olweus, D. (1998). Conductas de acoso y amenazas entre escolares. Madrid: Morata.

Padilla, J. (2006). De matones y matoneados. Una perspectiva desde el psicoanálisis. Bogota, Colombia: Cargraphis.

Pardo, M., Pineda, S., Carrillo, S., \& Castro, J. (Septiembre - Diciembre de 2006). Análisis psicométrico del inventario de apego con padres y pares en una muestra de adolescentes Colombianos. Interamerican Journa of Psychology, 40(3), 289-302. Obtenido de: http://www.redalyc.org/ pdf/284/28440304.pdf

Rozenel, V. (2006). Los modelos operativos internos (IWM) dentro de la teoría del apego. Aperturas psicoanalíticas. Obtenido de: $h t t p: / / w w w . a p e r t u r a s$. org/articulos. php?id=0000404

Santamaría, B. (Diciembre de 2013). Estilo educativo parental asociado al rol de víctimas en el fenómeno del Bullying. Revista Electrónica de Psicología Social «Poiésis», 1-16. Obtenido de: http://www.funlam.edu.co/revistas/ index.php/poiesis/article/view/997/888

Spitz, R. (1994). Introducción Teórica. El Primer Año de vida del niño. Bogotá: Fondo de Cultura Economica, Ltda.

Urizar, M. (2012). El vínculo afectivo y sus trastornos. Bilbao: CSMIJ. Obtenido de: http://www.avpap.org/documentos/bilbao2012/DesarrolloAfectivoAVPap. pdf

Varela, J., \& Lecannelier, F. (2008). Violencia Escolar (Bullying): ¿Qué es y cómo intervenir? Centro de Estudios Evolutivos e Intervención en el Niño. Obtenido de: https://es.scribd.com/doc/18167272/Documento-BullyingCEEIN-2009

Winnicott, D. (1990). Los bebés y sus madres. España: Paidós

Yáñez, J. (2012). 2012) Experticia, sabiduría y desarrollo Moral. Bogotá. (J. Chaparro, \& L. Segovia, Edits.) Bogotá DC [co]: Ed. Corporación Universitaria Minuto de Dios.

Zabarain, S., \& Sánchez, D. (24 de Noviembre de 2009). Implicaciones del Bullying o maltrato entre pares en el desarrollo psicoafectivo de niños y niñas en etapa de latencia. Psicogente, 12(22), 407-421. Obtenido de: $h t t p: / /$ revistas.unisimon.edu.co/index.php/psicogente/article/view/1174/1161

Zurita, Ú. (24 de Diciembre de 2011). Los desafíos del programa escuela segura. Revista Rayuela, Revista Iberoamericana sobre niñez y juventud en lucha por sus derechos, 1(2), 118-124. Obtenido de: http:// revistarayuela.ednica.org. mx/article/los-desaf\%C3\%ADos-del-programa\%E2\% $80 \% 9$ Cescuela-segura\%E2\%80\%9D-en-el-vig\%C3\%A9simoaniversario-de-la-convenci\%C3\%B3n-sobre 